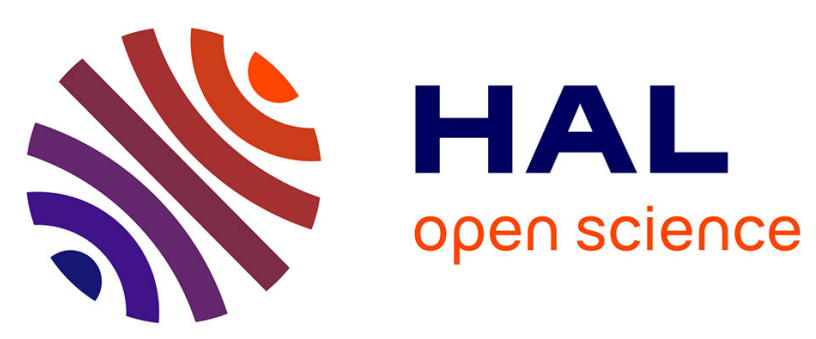

\title{
PHOTOEMISSION OVERVIEWS OF VALENCE BAND DENSITIES-OF-STATES FOR Ge, GaAs, GaP, InSb, ZnSe AND CdTe USING SYNCHROTRON RADIATION
}

D. Eastman, J. Freeouf, M. Erbudak

\section{To cite this version:}

D. Eastman, J. Freeouf, M. Erbudak. PHOTOEMISSION OVERVIEWS OF VALENCE BAND DENSITIES-OF-STATES FOR Ge, GaAs, GaP, InSb, ZnSe AND CdTe USING SYNCHROTRON RADIATION. Journal de Physique Colloques, 1973, 34 (C6), pp.C6-37-C6-44. 10.1051/jphyscol:1973610 . jpa-00215331

\section{HAL Id: jpa-00215331 https://hal.science/jpa-00215331}

Submitted on 1 Jan 1973

HAL is a multi-disciplinary open access archive for the deposit and dissemination of scientific research documents, whether they are published or not. The documents may come from teaching and research institutions in France or abroad, or from public or private research centers.
L'archive ouverte pluridisciplinaire HAL, est destinée au dépôt et à la diffusion de documents scientifiques de niveau recherche, publiés ou non, émanant des établissements d'enseignement et de recherche français ou étrangers, des laboratoires publics ou privés. 


\title{
PHOTOEMISSION OVERVIEWS OF VALENCE BAND DENSITIES-OF-STATES FOR Ge, GaAs, GaP, InSb, ZnSe AND CdTe USING SYNCHROTRON RADIATION $\left({ }^{*}\right)$
}

\author{
D. E. EASTMAN
}

IBM T. J. Watson Research Center, Yorktown Heights, N, Y 10598, USA

and

\section{J. FREEOUF and M. ERBUDAK}

Division of Engineering and Applied Physics, Harvard University, Cambridge, Mass. 02138, USA

\begin{abstract}
We present photoemission overviews of major valence band features for the valence bands of several common group IV, III-V and II-VI semiconductors, which were obtained using synchrotron radiation in the $20-90 \mathrm{eV}$ range from the $2.5 \mathrm{GeV}$ storage ring at the Cambridge Electron Accelerator. Comparisons of our photoemission data with empirical pseudopotential method (EPM) calculations fit to optical data show systematic and significant differences which typically increase with increasing ionicity. InSb is described in some detail, including measurements of core level binding energies, spin-orbit splittings and Auger processes. Several precautions when interpreting valence band photoemission measurements in the 20 to $90 \mathrm{eV}$ photon energy range are discussed (e. g. Auger processes, surface preparation, small matrix elements).
\end{abstract}

1. Introduction. - We describe photoemission measurements for several common group IV, III-V and II-VI semiconductors which have been obtained in the $20-90 \mathrm{eV}$ photon energy range using synchrotron radiation. From these measurements we have obtained overviews of the valence bands' optical density of states and have determined several valence band edges or critical points for each semiconductor with a typical precision of about 0.2 to $0.3 \mathrm{eV}$ with respect to the upper valence band edge $E_{v}$ [1], [2].

The current understanding of the electronic structure of semiconductors away from the gap has been obtained primarily by interpreting optical (including modulation) measurements via model band calculations [3], [4] such as the empirical pseudopotential method [3] (EPM). This procedure, which determines differences in band energies but usually not absolute band energies is a sensitive probe of the upper valence bands and lowest conduction bands. Previously, ultraviolet photoemission measurements for $h v \leqslant 11.6 \mathrm{eV}$ (LiF window cutoff) have proved useful for deter-

$\left({ }^{*}\right)$ Supported in part by the Air Force Office of Scientific Research under Contract F44620-70-C-0089, the National Science Foundation under Contract GH-35823 and the Atomic Energy Commission under Contract AT (11-1) 3063. mining certain valence band features within $\sim 4 \mathrm{eV}$ of the valence band edge [5].

Our measurements provide additional band information, including the lower valence bands [5] which, combined with optical data, should provide a guide for refining and testing local and non-local empirical pseudo-potential models as well as effective oneelectron potentials in «first-principles »calculations such as OPW or APW calculations. Our data at high photon energies ( $h v \gtrsim 30-50 \mathrm{eV}$ ) are quite similar to recent $X$-ray photoemission data $(h v \simeq 1500 \mathrm{eV})$, which are also proving to be very useful in determining valence band features [6]. This close similarity is seen even though the bulk penetration depth, e. g. electron escape depth, is considerably shorter in the $20-90 \mathrm{eV}$ range than at $1500 \mathrm{eV}$.

We use InSb as an example to discuss the trend from the UV photo-emission regime (roughly $h v \lesssim 20 \mathrm{eV}$ ) where both energy and momentum conservation are important to the «X-ray limit» ( $h v \gtrsim 30-50 \mathrm{eV}$ ) where only energy conservation is important, i. e. where photoemission spectra reflect single-particle density of states features. Various precautions when interpreting photoemission data in the $20-90 \mathrm{eV}$ range are offered... 
The use of intense synchrotron radiation has permitted us to obtain high resolution ( $\sim 0.1-0.4 \mathrm{eV})$ in the $10-100 \mathrm{eV}$ range. For example, for $\mathrm{InSb}$ we have determined $\mathrm{In}$ and $\mathrm{Sb} 4 \mathrm{~d}$ core level binding energies

$$
\begin{aligned}
\left(I n: d_{5 / 2}\right. & =17.40 \pm 0.05 \mathrm{eV} \\
\mathrm{Sb}: \mathrm{d}_{5 / 2} & =32.52 \pm 0.05 \mathrm{eV})
\end{aligned}
$$

spin-orbit splittings

$$
\begin{aligned}
(\text { In }: \Delta & =0.85 \pm 0.03 \mathrm{eV}, \\
\mathrm{Sb}: \Delta & =1.25 \pm 0.03 \mathrm{eV})
\end{aligned}
$$

and line widths

$$
\begin{aligned}
& (\text { In : } 2 \Gamma=0.48 \pm 0.03 \mathrm{eV} \\
& \text { Sb : } 2 \Gamma=0.62 \pm 0.05 \mathrm{eV})
\end{aligned}
$$

Various trends can be seen in our valence band overviews. For example, we observe that the upper three valence bands narrow with increasing ionicity, i. e. on going from group IV (Ge) to group III-V (GaAs, GaP, InSb) to group II-VI ( $\mathrm{ZnSe}, \mathrm{CdTe}$ ) semiconductors. Also, as theoretically expected [3], the lowest (first) valence band is seen to split off from the upper three valence bands for the heteropolar semiconductors (e. g. GaAs, etc.). EPM calculations fit to optical data consistently obtain widths for the upper three valence bands which are narrower than we observe, and in the case of the II-VI semiconductors ( $\mathrm{ZnSe}, \mathrm{CdTe}$ ), these differences become quite large. For example, in GaAs the upper three valence bands $\left(E_{v}-X_{3}\right)$ are $6.9 \mathrm{eV}$ wide (experiment) while the EPM [8] yields $6.4 \mathrm{eV}$ while in $\mathrm{ZnSe}$ the corresponding widths are $5.3 \mathrm{eV}$ (experiment) and $3.8 \mathrm{eV}$ (EPM) [8]. Typically, OPW calculations show somewhat better agreement with our data for the lower valence bands than EPM calculations fit to optical data [9], [12]. Recently, various workers have modified EPM calculations via the inclusion of non local potentials and obtain much better agreement with experiment [13], [15].

2. Experimental. - A schematic diagram of the experimental system at the $2.5 \mathrm{GeV}$ Cambridge Electron Accelerator is shown in figure 1. An ellipsoidal focusing mirror with a $0.6^{\circ}$ grazing angle captures $10 \mathrm{mrad}$ of horizontal beam spread and focuses an image of the source point $(\sim 1 \times 2 \mathrm{~mm})$ in the storage ring into the user room about 20 meters from the source [16]. This mirror can be adjusted remotely and passes all wavelengths $\lambda$ greater than $\lambda_{c} \simeq 3.5 \AA$, i. e. $l h v_{\mathrm{c}} \simeq 3.5 \mathrm{keV}$. Radiation shielding permits occupancy of the user room during all phases of storage ring operation. With the storage ring operating at $2.5 \mathrm{GeV}$ and $30 \mathrm{~mA}$ of $\mathrm{d}$. c. beam current, the flux of focused radiation is about $10^{14}-10^{15}$ photons/eV .s in the $10-100 \mathrm{eV}$ range.

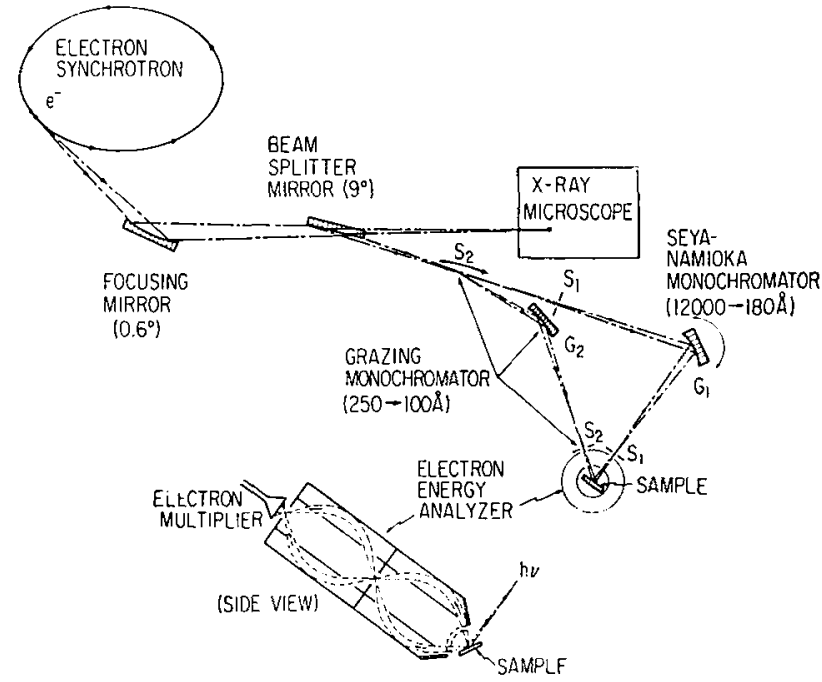

FIG. 1. - Schematic diagram of photoemission system using synchrotron radiation at the $\mathrm{CEA} 2.5 \mathrm{GeV}$ storage ring (not to scale). The storage ring is $\sim 80 \mathrm{~m}$ diameter and the experimental system is $\sim 20 \mathrm{~m}$ from the source.

A planar beam splitter mirror with a $2.5 \mathrm{~mm} \mathrm{dia-}$ meter hole is used to split the beam for simultaneous operation of an X-ray microscope [16] and our photoemission system. This mirror has a $9^{\circ}$ grazing angle and acts as a low pass filter, i. e. passes only radiation with $h v \lesssim 200-300 \mathrm{eV}$, so as to avoid X-ray damage to the Au-coated replica gratings in the monochromators.

Ultra-high-vacuum (UHV) monochromators

$$
\left(p \lesssim 1 \times 10^{-9} \text { torr }\right)
$$

have been constructed so as not to degrade the vacuum of the sample chamber ( $p \lesssim 3 \times 10^{-10}$ torr). Two monochromators with fixed exit slits have been built, a I-m Seya-Namioka type (slits $S_{1}$ and grating $\mathrm{G}_{1}$ in figure 1) useable in the $12,000-180 \AA$ range and a $2-m$ grazing incidence type (slits $\mathrm{S}_{2}$ and grating $\mathrm{G}_{2}$ ) useable in the $250-100 \AA$ range. In order to both maximize efficiency over a wide spectral range and to minimize unwanted higher order diffraction effects, the Seya-type monochromator has two gratings blazed at $\sim 1200 \AA$ and $300 \AA$, respectively which can be interchanged in situ. This arrangement, together with a LiF filter for wavelengths $\lambda>1060 \AA$, has proved satisfactory for most measurements. Adjustable slits permit the band width $\Delta \lambda$ to be varied from $\Delta \lambda=32 \AA$ to $1 \AA$ in the range $12,000-180 \AA$.

The 2-m grazing incidence monochromator consists of a fixed exit slit $\mathrm{S}_{2}$, fixed-position 2400 groove $/ \mathrm{mm}$ spherical grating blazed at $\sim 120 \AA$ and a moveable entrance slit which consists of a $0.15 \mathrm{~mm}$ wide planar mirror (Au coated) which translates and rotates along the Rowland circle in the path of the photon beam. The band width $\Delta \lambda$ can be varied from about $0.3 \AA$ to $4 \AA$ throughout the $100 \AA$ to $250 \AA$ range. 
The monochromatic beams of both monochromators pass through the focal point of the three-stage cylindrical mirror electron analyzer. Sampies can be positioned for use with either monochromator by a simple rotation. The cylindrical mirror analyzer consists of a retard/accelerate stage constructed from two concentric hemispherical grids centered about the sample, followed by a two-stage electrostatic deflection, $2^{\prime \prime}$ i, d. cylindrical mirror analyzer with a Bendix spiraltron electron muitiplier [17]. The passband width $\Delta E$ (typically 0.1 to $0.4 \mathrm{eV}$ ) is determined by selecting the pass energy $E_{\mathrm{p}}=\Delta E . R$ with a resolution $R \simeq 125$. This analyzer has spatial focusing which permits easy alignment to both monochromators, and operates quite well down to zero kinetic energy by pre-accelerating the emitted electrons using the inner hemispherical grid.

Single crystal semiconductors of $2 \mathrm{~mm} \times 2 \mathrm{~mm}$ cross section were cleaved (with some faceting) and measured in situ at about $3 \times 10^{-10}$ torr, with a (111) surface for $\mathrm{Ge}$ and (110) surfaces for the heteropolar semiconductors. Lightly-doped samples (except for $\mathrm{ZnSe}$ ) were used to avoid complications due to short bandbending depths.

3. Data and discussion. - 3.1 InSb. - We shall first describe InSb as a prototype, illustrating various features which can be determined as well as pointing out various precautions which should be observed in interpreting photoemission data.

A photoemission energy distribution (PED) for $h v=70 \mathrm{eV}$ is shown in figure 2 for InSb and gives an overview of both the valence bands and the $4 d$ core levels. In addition to primary emission from the valence bands and $4 \mathrm{~d}$ core levels, we observe several characteristic energy loss peaks (CEL) which accompany the core level lines as well as two broad Auger

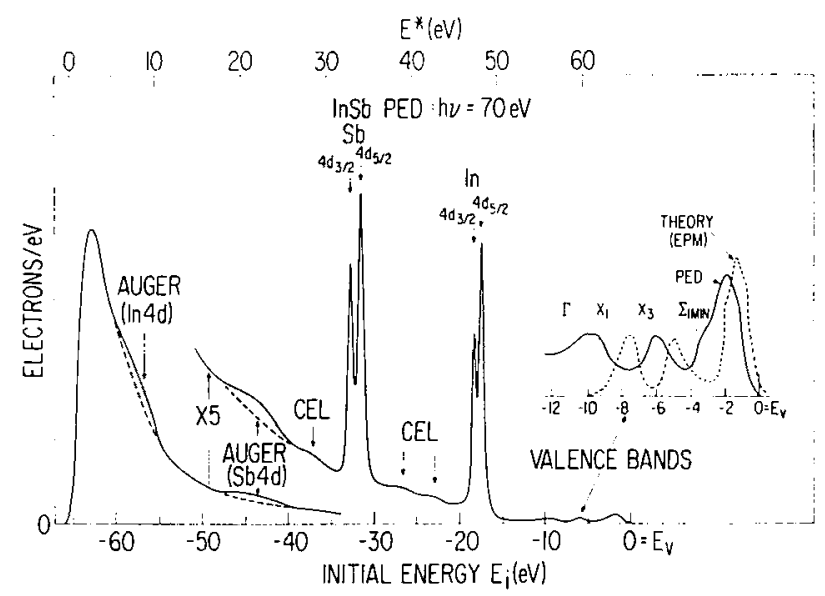

FIG. 2. - Photoemission energy distribution for InSb with $h v=70 \mathrm{eV}$. Photon and electron energy band widths of $\Delta h v \simeq \Delta E^{*} \simeq 0.38 \mathrm{eV}$ were used for the valence bands and $\Delta h v \simeq \Delta E^{*} \simeq 0.2 \mathrm{eV}$ for the core levels. The core level counting rates were $\sim 50,000 \mathrm{elec} / \mathrm{s}$ (for $\Delta h v \simeq \Delta E^{*} \simeq 0.4 \mathrm{eV} ; 30 \mathrm{~mA}$ beam). peaks which remain stationary in kinetic energy near $E^{*} \simeq 8 \mathrm{eV}$ and $25 \mathrm{eV}$. These Auger peaks result from $\mathrm{N}_{4.5} \mathrm{VV}$ transitions of the In $4 \mathrm{~d}$ and $\mathrm{Sb} 4 \mathrm{~d}$ core levels, respectively.

Considering first the valence bands, several PED's are shown in figure 3. These PED's were selected because they show various valence band features and also illustrate effects due to Auger emission and transition probabilities. Valence band energies are measured relative to the valence band edge $E_{\mathrm{v}}=0$. The valence band edge $E_{\mathrm{v}}$ was determined by measuring the Fermi level $E_{\mathrm{F}}$ of a gold film evaporated in situ; $E_{\mathrm{v}}$ for lightly-doped InSb was determined via a PED for $h v=8 \mathrm{eV}$ to lie $\sim 0.25 \mathrm{eV}$ below $E_{\mathrm{F}}$ [18].

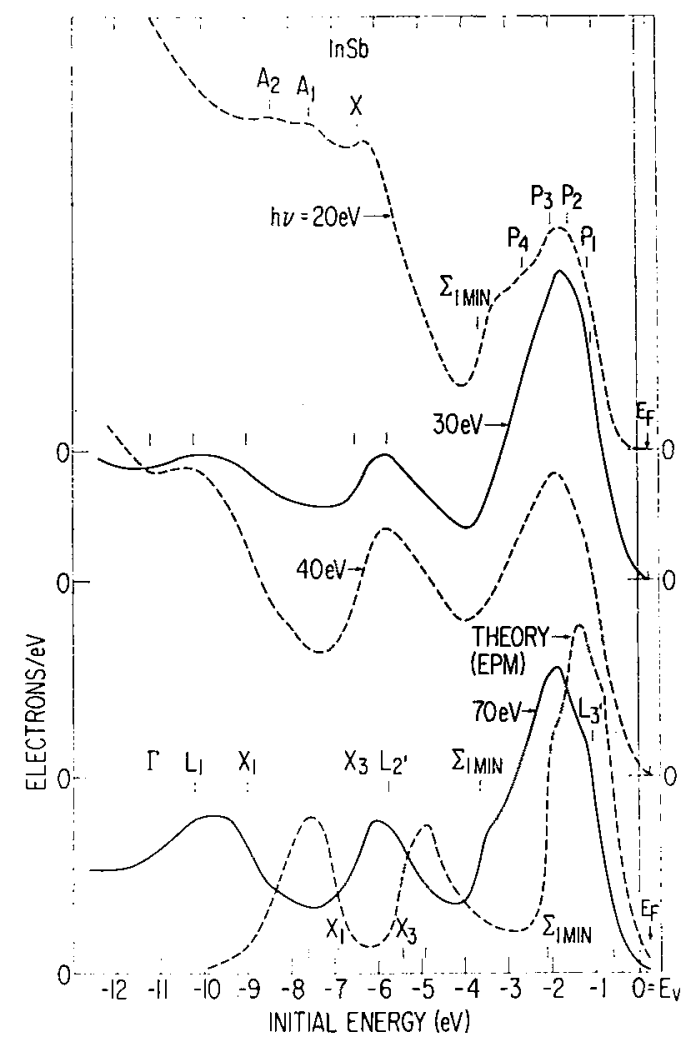

FIG. 3. - InSb valence band energy distributions for $h v=20$, 30, 40 and $70 \mathrm{eV}$. Experimental valence band critical points are marked above the $70 \mathrm{eV}$ curve. A theoretical density of states curve [10], [22] (empirical pseudopotential method) is also shown with critical points marked along the lower axis. The counting rates varied monotonically from $\sim 4 \times 10^{4} \mathrm{elec} / \mathrm{s}$ at $h v=20 \mathrm{eV}(\Delta h v \simeq \Delta E \simeq 0.2 \mathrm{eV})$ to $2 \times 10^{3} \mathrm{elec} / \mathrm{s}$ at $h v=70 \mathrm{eV}(\Delta h v \simeq \Delta E \simeq 0.4 \mathrm{eV})$ with a beam current of $\simeq 30 \mathrm{~mA}$.

The PED for $h v=20 \mathrm{eV}$ shows the width of the valence p-bands (bands 3 and 4 ), i. e

$$
E_{\mathrm{v}}-\Sigma_{1 \min }=3.65 \mathrm{eV},
$$

as well as additional structures due to primary photoelectron emission (features $\mathrm{P}_{1}-\mathrm{P}_{4}$ and $X_{3}$ ) and to Auger electron emission $\left(A_{1}, A_{2}\right)$. While feature $\mathrm{P}_{1}$ corresponds to the $L_{3}$ edge seen at higher $h v$ 's, features 
TABLE I

Positions of energy band features measured relative to the valence band edge (eV). Theoretical EPM values are given in parenthesis (see ref. [22])

\begin{tabular}{|c|c|c|c|c|c|c|}
\hline & $L_{3}$ & $\Sigma_{1 \text { min }}$ & & $L_{1}$ & $L_{2}$ & $\Gamma_{1}$ \\
\hline $\mathrm{Ge}$ & $1.1 \underset{L_{3}}{(1.1)}$ & $\underset{\Sigma_{1 \mathrm{~min}}}{4.5}$ & 7.7 & $X_{3}^{(6.9)}$ & $\begin{array}{c}10.6(9.9) \\
X_{1}\end{array}$ & $\begin{array}{c}12.6(12.0) \\
\Gamma_{1}\end{array}$ \\
\hline GaAs & $0.8(0.9)$ & 4.1 (3.2) & 6.9 & a) $(6.4)$ & $10.0(9.7)$ & $12.9(12.0)$ \\
\hline $\mathrm{GaP}$ & $0.8 \quad(0.9)$ & $4.1 \quad(3.8)$ & 6.9 & (5.8) & $9.7(11.5)$ & $11.8(13.0)$ \\
\hline $\mathrm{InSb}$ & $1.05(0.6)$ & $3.65(2.1)$ & 6.5 & (5.4) & $9.0(7.6)$ & $11.2(8.9)$ \\
\hline $\mathrm{ZnSe}$ & $0.7 \quad(0.45)$ & $3.4 \quad(2.7)$ & 5.3 & (3.8) & - & $-(14.2)$ \\
\hline $\mathrm{CdTe}$ & $0.7 \quad(0.4)$ & $2.8 \quad(2.0)$ & 4.7 & (2.9) & $8.8(12.5)$ & $-(12.9)$ \\
\hline
\end{tabular}

$\left({ }^{a}\right)$ See footnote 25

$\mathrm{P}_{2}-\mathrm{P}_{4}$ vary with photon energy for $h v \lesssim 20 \mathrm{eV}$. This behavior is characteristic of momentum conservation in the excitation process at lower energies [2]. The weak but well-defined feature $X_{3}$ corresponds to the bottom of band 2 (this critical point is best seen at $h v \simeq 15 \mathrm{eV}$ ).

Interestingly, no emission from the lowest valence band (band 1) centered near $-10 \mathrm{eV}$ is seen for $h v \leqslant 25 \mathrm{eV}$. Emission from this lowest Sb-derived band is seen for $h v=30 \mathrm{eV}$ and increases relative to p-bands 3 and 4 with increasing $h v$ (e. g. see PED for $h v=70 \mathrm{eV}$ ). We have observed a similar behavior for several semiconductors (e. g. GaAs and GaP). This serves as a warning that a PED at a fixed energy such as $21 \mathrm{eV}$ in general will not reflect all valence bands, i. e. bands can be « missing ». Also for $h v=20 \mathrm{eV}$, Auger emission due to In $4 \mathrm{~d}$-holes overlaps valence band emission and results in intense emission for initial energies $E_{\mathrm{i}} \lesssim-5 \mathrm{eV}$, with Auger structure observed at -7.5 and $-8.5 \mathrm{eV}\left(A_{1}, A_{2}\right)$. Thus, care must be used in interpreting observed features when Auger emission is possible. Such Auger emission does not interfere with valence band emission for $h v=30 \mathrm{eV}$, as is seen in figure 3 .

The PED for $h v=30 \mathrm{eV}$ reflects many valence band density of states features, including the $L$ edge $L_{3}$ of band 4 , features $L_{2}$ and $X_{3}$ of band 2 and features $X_{1}, L_{1}$ and $\Gamma_{1}$ of band 1 . However, the minimum of band $3\left(\Sigma_{1 \mathrm{~min}}\right)$ is not clearly seen for $h v=30 \mathrm{eV}$ (or for $h v=40 \mathrm{eV})$. Comparing PED's for $h v=40 \mathrm{eV}$ and $30 \mathrm{eV}$, at $h v=40 \mathrm{eV}$, emission from bands 1 and 2 has become more intense relative to that from bands 3 and 4 . However at $40 \mathrm{eV}$, increased emission for initial energies $E_{\mathrm{i}} \lesssim-10 \mathrm{eV}$ is also observed due to Auger emission from the $\mathrm{Sb} 4 \mathrm{~d}$-holes.

At higher energies, e. g. at $h v=70 \mathrm{eV}$, the « X-ray limit» (or continuum limit) is reached and valence band density-of-states features are reflected in the PED. Valence band features (i. e. critical points) observed for this PED as well as other PED's are indicated by the vertical dashes. These energy levels are summarized in table I and were determined by comparison with suitably-broadened theoretical singleparticle density of states due to Alvarez, et al. [19], for which the corresponding critical points are known [2]. The disagreement between our experimental values and the EPM values [19] is not unexpected in that the latter bands were fit only to dielectric constant data, involving mainly valence band 4 . As seen in figure 3 the EPM bands are too narrow, and have a much smaller separation $\left(X_{3}-X_{1}\right)$ between the first and second valence bands than is observed. Unadjusted OPW calculations [9], [20] (see Table I) give a better fit to these lower valence bands. It should be interesting to see how well such calculations can fit both optical data and our valence band data.

Considering now the $4 \mathrm{~d}$ core levels of $\operatorname{InSb}$, the high resolution spectrum in figure 2 clearly shows the spin-orbit splittings and level widths of the In $4 \mathrm{~d}$ and $\mathrm{Sb} 4 \mathrm{~d}$ levels. These binding energies (measured relative to the Fermi level $E_{\mathrm{F}}$ which is at the conduction band minimum $E_{\mathrm{c}}$ ), splittings, and level widths are summarized in table II. For comparison, we have measured the $4 d$ levels in metallic In (Table II). Comparing InSb and In metal, a chemical shift of $0.70 \mathrm{eV}$ is observed [20] for the In $4 \mathrm{~d}$ level. Also, our measured In $4 d$ level width (FWHM $2 \Gamma$ ) is greater for $\operatorname{InSb}(2 \Gamma=0.48 \pm 0.03 \mathrm{eV})$ than for In metal $(2 \Gamma=0.37 \pm 0.03 \mathrm{eV})$.

In our photoemission studies of semiconductors

\section{TABLE II}

InSb core levels ( $E_{\mathrm{F}}$ reference). The Fermi level $E_{\mathrm{F}}$ is $\approx 0.25 \mathrm{eV}$ above the valence band edge $E_{\mathrm{v}}$.

\begin{tabular}{lll}
\multicolumn{1}{c}{ Level } & Energy $(\mathrm{eV})$ & Width $2 \Gamma(\mathrm{eV})$ \\
In $4 \mathrm{~d}_{5 / 2}$ & $17.40 \pm 0.05$ & $0.48 \pm 0.03$ \\
$4 \mathrm{~d}_{3 / 2}$ & 18.25 & $\left(\Delta_{\mathrm{s} 0}=0.85\right)$ \\
$\mathrm{Sb} 4 \mathrm{~d}_{5 / 2}$ & 31.52 & $0.62 \pm 0.05$ \\
$4 \mathrm{~d}_{3 / 2}$ & 32.77 & $\left(\Delta_{\mathrm{s} 0}=1.25\right)$ \\
In metal $4 \mathrm{~d}_{5 / 2}$ & 16.70 & $0.37 \pm 0.03$ \\
$4 \mathrm{~d}_{3 / 2}$ & 17.58 & $\left(\Delta_{\mathrm{sc}}=0.88\right)$
\end{tabular}


in the $10-100 \mathrm{eV}$ range, we have observed that surface preparation, both structural preparation and surface cleanliness, is very important, especially for the more covalent materials Si, Ge, GaAs, etc. We have observed greater surface sensitivity for $20 \mathrm{eV} \lesssim h v \lesssim 40 \mathrm{eV}$ than for $h v \lesssim 15 \mathrm{eV}$ or $h v \gtrsim 50 \mathrm{eV}$. This is consistent with the shortest electron escape depth occurring at about twice the plasma energy $f i \omega_{\mathrm{p}} \sim 15 \mathrm{eV}$, as is given by free electron gas calculations.

We next consider photoemission data for Ge, GaAs, $\mathrm{GaP}, \mathrm{ZnSe}$ and $\mathrm{CdTe}$ and give brief descriptions of various valence band features. Photoemission energy distributions (PED's) have been selected at sufficiently high energies to give overviews of main valence band features. Experimental valence band «photoemission densities of states» (PDS's) and previously reported theoretical band densities of states determined via the empirical pseudopotential method are also presented. The PDS's are derived from the experimental PED's by subtracting a smooth background of inelastically scattered secondary electrons [2]. As discussed for InSb, principal features in these « high energy » PED's at photon energies which avoid interference from Auger emission are expected to correspond to structure in the valence band density of states.

3.2 Germanium. - A photoemission energy distribution curve for $\mathrm{Ge}$ is shown in figure $4 a$ and the corresponding photoemission density of states (PDS) together with an EPM density of states $N(\mathrm{E})$ is shown in figure $4 b$ [22]. The EPM curve is broadened by a Lorentzian of full-width $2 \Gamma$ varying linearly with energy from $0.2 \mathrm{eV}$ at the valence band edge $E_{\mathrm{v}}$ to

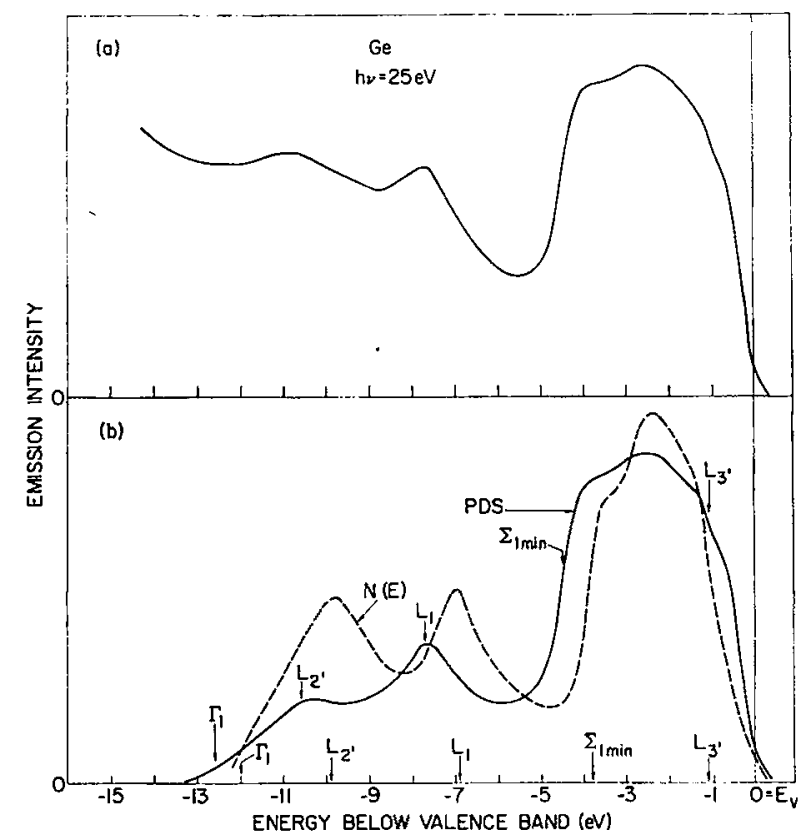

FIG. 4. - (a) Photoemission energy distribution for Ge. (b) « Photoemission density of states » (PDS) and EPM density of states $N(E)$. Experimental critical points (upper set) and theoretical critical points (lower set) are marked. See reference [22].
$0.75 \mathrm{eV}$ at $E_{\mathrm{v}}-12 \mathrm{eV}$ in order to roughly simulate intrinsic lifetime broadening and experimental broadening [24].

Our experimental valence band edges and critical points for Ge are summarized in table I together with corresponding EPM values. All values are measured relative to the valence band edge $E_{\mathrm{v}}$. Experimental band edges and other critical points were determined by comparing with corresponding features in the "lifetimes-broadened" EPM calculations for which the corresponding critical points are known [22] (lower arrows in figure $4 b$ ). Considering that the EPM results were not fit to the lower valence bands, the overall shape of the EPM valence band density of states for $\mathrm{Ge}$ is in good agreement with our PDS, and the disagreement in position of the lower bands is not surprising. The enhanced edge just below $E_{\mathrm{v}}$ in our PDS is believed to be due to intrinsic surface states [19]. The presence of these surface states might also slightly affect our assignment of the $L_{3}$ edge $(1.1 \mathrm{eV})$. Experimentally, the most accurately determined features are the low energy features of the second and third valence bands $\left(L_{1}\right.$ and $\left.\Sigma_{1 \min }\right)$ and EPM calculations (as well as OPW calculations) predict band widths which are generally too narrow by $\sim 0.5 \mathrm{eV}$, or more. Recently, an EPM calculation which includes a non local d-phase shift has been reported [13] which fits our experimental edges very well.

3.3 Gallium Arsenide. - A photoemission energy distribution curve is shown in figure $5 a$ together with the corresponding PDS and an EPM density of states $N(\mathrm{E})$ in figure $5 b$. Our experimental valence

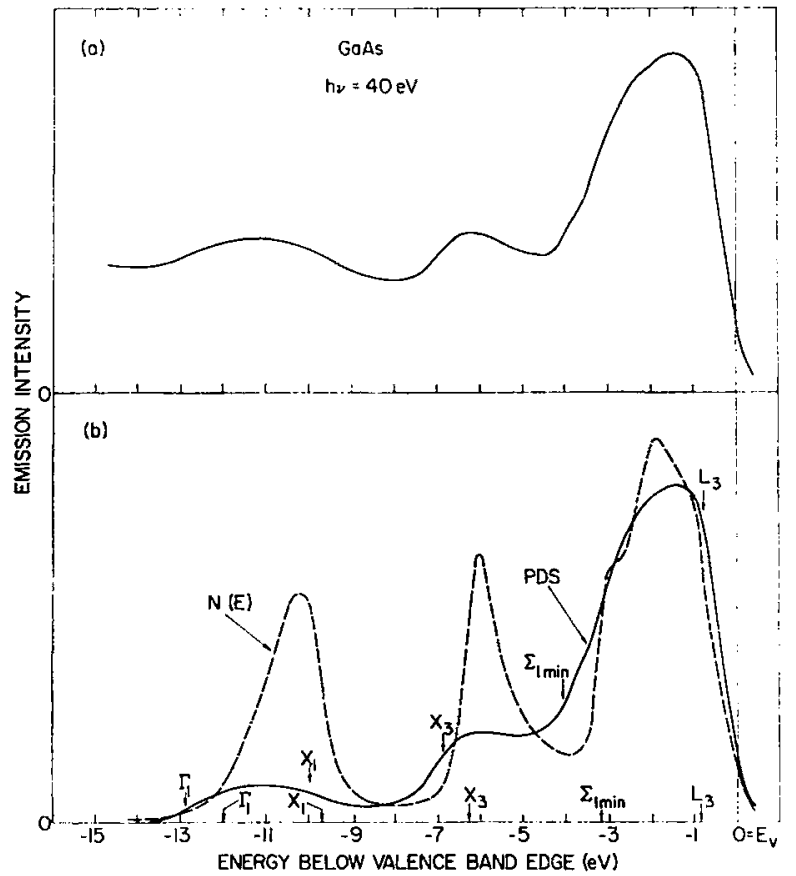

Fig. 5. - (a) Photoemission energy distribution for GaAs. (b) «Photoemission density of states" (PDS) and EPM density of states $N(\mathrm{E})$ [23]. 
band edges (critical points) are shown in figure $5 b$ (upper arrows) and are summarized in table I. For GaAs (as for the other semiconductors) the assignments for $\Sigma_{1 \min }, X_{3}$, etc., were checked and made more precise by reference to PED's for other photon energies. Such lower energy PED's show the $\Sigma_{1 \text { min }}$ and $X_{3}$ edges with better resolution but do not directly reflect other density of states features (due to momentum conservation) and cannot give an overview of the entire valence band. The valence band edges $\Gamma_{1}$ and $X_{1}$ for band 1 were determined from a PED for $h v=70 \mathrm{eV}$ which better shows this lowest valence band.

The EPM density of states fit to optical data [8] gives a good overview of the upper three yalence bands. However, the EPM band edges $X_{3}$ and $\Sigma_{1 \mathrm{~min}}$ of the second and third bands are too high by $0.5 \mathrm{eV}$ and $0.9 \mathrm{eV}$ respectively [25]. Interestingly, the EPM model shown in figure $5 b$, which represents a refinement [8] of an earlier model [7] in that optical modulation data were also fit, shows poorer agreement with our data in the position of certain band edges such as $\Sigma_{1 \text { min }}$ than the earlier calculation.

3.4 Gallium Phosphide. - Photoemission energy distributions (PED's) for photon energies of $h v=20 \mathrm{eV}$ and $78 \mathrm{eV}$ are shown in figure $6 a$. Our resulting PDS is shown in figure $6 b$ together with an EPM density of states $N(E)$ [22]. Experimental critical points $L_{3}, \Sigma_{\min }, X_{3}, X_{1}$ and $\Gamma_{1}$ are shown by the upper arrows in figure $6 b$ and are summarized in table I. As seen in figure $6 a$, the PED for $h v=20 \mathrm{eV}$ more clearly shows the band edge critical points

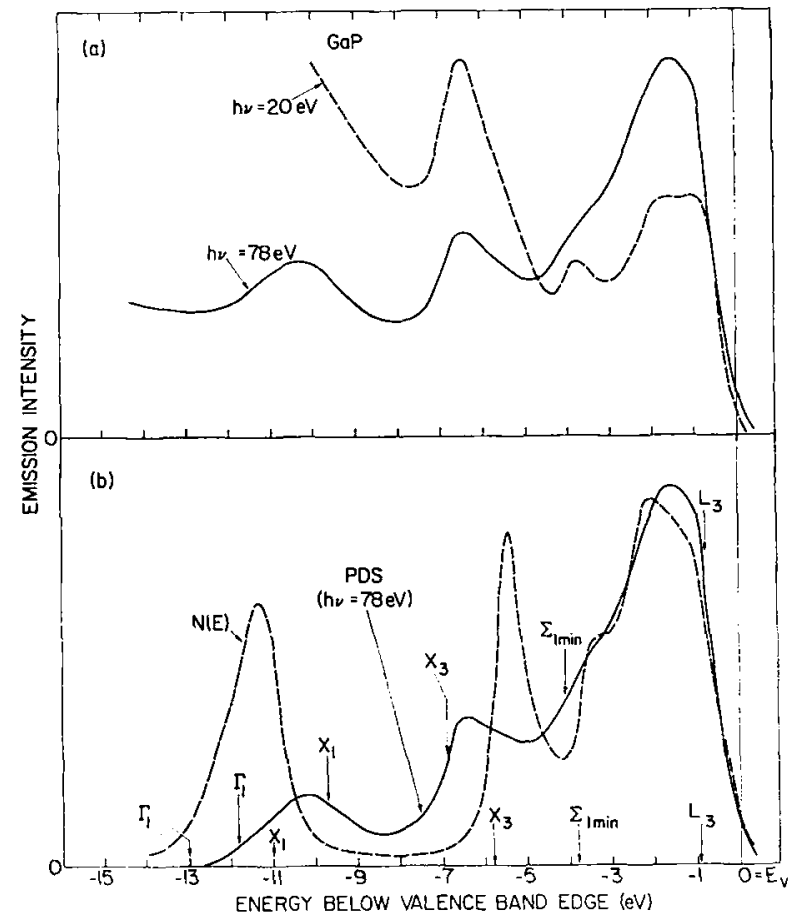

FIG. 6. - (a) Photoemission energy distributions for GaP. (b) «Photoemission density of states » (PDS) and EPM density of states $N(\mathrm{E})$ [8].
$\Sigma_{1 \min }$ and $X_{3}$, while the PED for $h v=78 \mathrm{eV}$ gives a much better overview of all 4 valence bands.

Comparing our experimental PDS's for GaP and GaAs with the corresponding EPM densities of states, the width of the upper two valence bands $\left(\Sigma_{1 \text { mill }}\right)$ shows better agreement for GaP than for GaAs. This is probably due to the fact that, in contrast with GaAs, GaP is an indirect-gap III-V semiconductor, and both the indirect gap $X_{1}$ (conduction band). $E_{\mathrm{v}}\left(\Gamma_{15}\right)$ and the $X_{1}-X_{5}$ band separations have been determined from the optical data. This determines the $E_{\mathrm{v}}-X_{5}$ separation, which is proportional to the width $\Sigma_{1 \min }$ of the upper two valence bands.

3.5 Zinc Selenide. - Photoemission energy distributions for $h v=20 \mathrm{eV}$ and $30 \mathrm{eV}$ are shown in figure $7 a$. Our resulting PDS is shown in figure $7 b$ together with an EPM density of states [22] $N(E)$. Comparing the PED's for $h v=20$ and $30 \mathrm{eV}$ in figure $7 a$, a large change in the spectral shape of the emission from the upper three valence bands is observed. The $\mathrm{Zn} 3 \mathrm{~d}$ bands centered at $8.9 \mathrm{eV}$ below $E_{\mathrm{v}}$ are also shown for the $30 \mathrm{eV}$ PED. For photon energies $h v \leqslant 40 \mathrm{eV}$, we do not see emission from the lowest valence band (Se-derived).

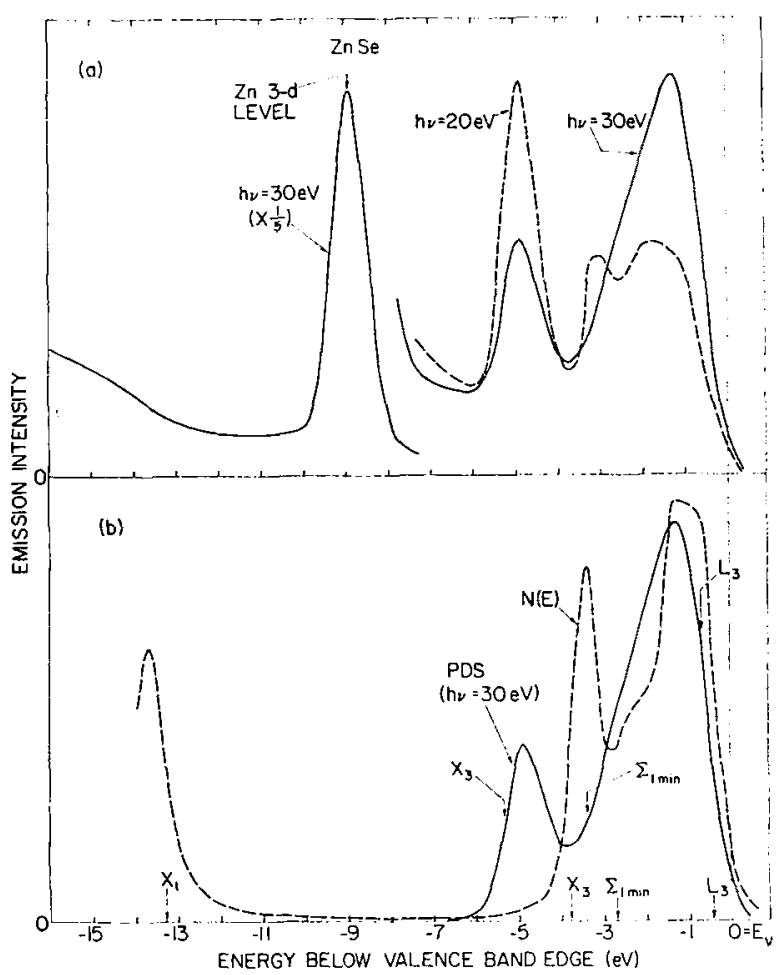

FIG. 7. - (a) Photoemission energy distributions for $\mathrm{ZnSe}$ (b) «Photoemission density of states " (PDS) and EPM density of states $N(\mathrm{E})$ [8].

Our experimental critical points for the upper three valence bands are summarized in table I. The band edges $\left(X_{3}=5.3 \pm 0.3 \mathrm{eV}\right.$ and $\left.\Sigma_{1 \mathrm{~min}}=3.4 \pm 0.3 \mathrm{eV}\right)$ of the second and third valence bands, respectively, obtained from our data are seen to be much deeper than the corresponding EPM values [8].

3.6 Cadmium Telluride. - A photoemission 
energy distribution for $h v=24 \mathrm{eV}$ is shown in figure $8 a$ and the corresponding PDS is shown in figure $8 b$, together with an EPM density of states [22]. Experimental valence band features are again summarized in table $\mathrm{I}$.

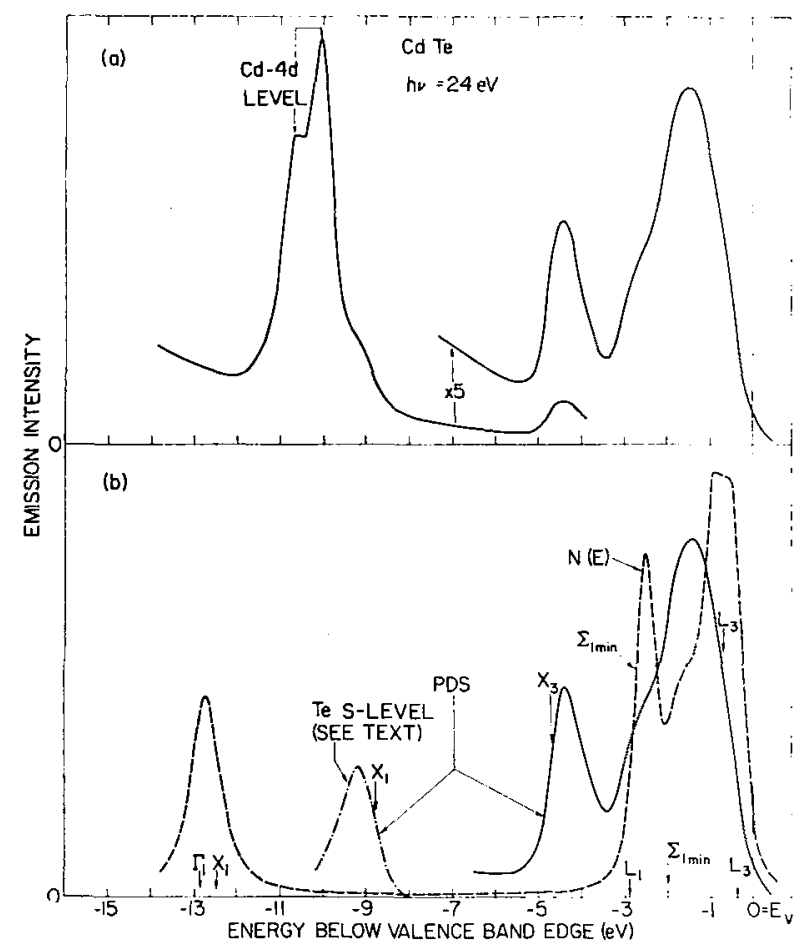

Fig. 8. - (a) Photoemission energy distribution for CdTe. (b) «Photoemission density of states » (PDS) and EPM density of states $N(\mathrm{E})$ [12].

As with $\mathrm{ZnSe}$, the widths of the second and third valence bands (e. g. $X_{3}=4.7 \pm 0.2 \mathrm{eV}$ and $\Sigma_{1 \min }=2.8 \pm 0.2 \mathrm{eV}$ ) are seen to be much broader than the EPM results $\left(X_{3}=2.9 \mathrm{eV}\right.$ and $\left.\Sigma_{1 \mathrm{~min}}=1.9 \mathrm{eV}\right)$. Also the gap between the first and second bands $\left(X_{3}-X_{1}=4.1 \mathrm{eV}\right)$ is much smaller than the EPM results $\left(X_{3}-X_{1}=9 \mathrm{eV}\right)$. This spectroscopically determined band gap has recently been shown to be a good measure of the ionicity parameter $C$ of the Phillips-Van Vechten theory [26].

The large discrepancy in $X_{3}-X_{1}$ in the EPM appears to be due to the sensitivity of the lowest band to the pseudopotential coefficients and the previous lack of experimental data for the lower bands; however, recent EPM calculations indicate that a non local pseudopotential is required to describe the lower bands [14], [15].

In figure $8 a$ we also observe the spin-orbit split $\mathrm{Cd} 4 \mathrm{~d}$ levels, at $-10.0 \mathrm{eV}\left(\mathrm{D}_{5 / 2}\right)$ and $-10.6 \mathrm{eV}$ $\left(D_{3 / 2}\right)$, respectively, which overlap the first valence band (mostly Te $5 \mathrm{~s}$ level) that has an edge $X_{1}$ at $8.8 \pm 0.3 \mathrm{eV}$. The bottom of the valence bands $\Gamma_{1}$ is roughly estimated as $10.8 \pm 0.6 \mathrm{eV}$; this estimate is based on the position of the $X_{1}$ edge and typical broadening of such deep bands (a lower bound of $\Gamma_{1}<11.5 \mathrm{eV}$ is obtained by inspection of the PED). Comparison with OPW calculations shows that unadjusted OPW calculations [11] give a more accurate picture of the lower valence bands than the EPM fit to optical data [12].

4. Summary. - We have presented overviews of the valence band structure for several common group IV, III-V and II-VI semiconductors. While overall shapes (but not amplitudes) of our photoemission densities of states are in qualitative agreement with EPM and OPW densities of states, we generally find significant differences in band edges and critical points for the lowest three valence bands. Ge, GaAs and $\mathrm{ZnSe}$ share the same row in the periodic table and show a systematic narrowing of valence bands 2 , 3 and 4 as one goes from Ge to more ionic $\mathrm{ZnSe}$. This trend is also seen in the EPM results as well as in OPW calculations [4]. However, the EPM bands are always narrower than experiment, with the discrepancy becoming significantly larger for II-VI $\mathrm{ZnSe}$ and CdTe. While it is beyond the scope of this paper to compare all available band calculations (OPW, etc.) with various one-electron potentials, we note that unadjusted OPW calculations [9], [11], [20] generally give a better description of the lower valence bands than do EPM calculations fit only to optical data.

Experimental valence band data such as presented in table I, used together with optical data, should provide a valuable guide to future band calculations e. $g$, in ascertaining the adequacy of various effective one-electron potentials in first-principles band calculations (OPW, APW) and in inquiring whether local pseudopotentials can fit semiconductor energy bands over a wide energy range or whether non-local corrections are required.

Photoemission overviews of valence band structure such as we have presented are in a preliminary stage and future work is expected to furnish additional valence band information as well as data for new materials. Recently, good overviews of valence band structure for semiconductors have been obtained via X-ray photoemission measurements [6]. Once overviews of valence band structure for semiconductors are established, then calculations of lower energy PED's $(h v \lesssim 25 \mathrm{eV})$, which show structure in the joint density of states, should become more useful and hopefully will yield much additional information about both valence and conduction bands. Such photoemission measurements and interpretations for semiconductors have yielded much information in the past [5], [27], mainly for $h v \lesssim 11.6 \mathrm{eV}$ and for the upper valence bands, and are expected to yield additional information at higher energies which have become available with the use of synchrotron radiation sources.

5. Acknowledgments. - The authors gratefully acknowledge the many contributions of D. J. Chadi, M. L. Cohen, W. D. Grobman, W. Paul and the CEA staff to this work. 


\section{References}

[1] This paper is an extension of an earlier paper on $\mathrm{Si}, \mathrm{Ge}$ and GaAs (Ref. 2) which described the technique.

[2] Grobman, W. D. and Eastman, D. E., Phys. Rev. Lett. 29 (1972) 1508.

[3] A comprehensive review of the EPM is presented by Conen, M. L. and Heine, V., Solid State Physics, Vol. 24, edited by H. Ehrenreich, F. Seitz and D. Turnbull (Academic Press, New York 1970), p. 38-249.

[4] Empirically adjusted OPW calculations are described in : Herman, F., et al. in Methods in Computational Physics, Vol. 8, edited by B. Alder, S. Fernbach and M. Rotenberg (Academic Press, New York, 1968), p. $193-250$.

[5] Previous experiments have usually been confined to bands near the gap by the LiF window cutoff $h v \lesssim 11.6 \mathrm{eV}$. Reviews of previous experimental work are given in Spicer, W. E. and Eden, R. C., Proc. IX Int. Semiconductor Conf. (1968), Vol. 1, p. 61 ; and SPICER, W. E., J. of Research (NBS) 74A (1969) 397.

[6] Ley, L., et al., Phys. Rev. Lett. 29 (1972) 1088 ; Pollak, R. A., et al., ibid 29 (1972) 1103 ; MCFEELY, F. R., et al., Phys. Rev. B, April 15 (1973).

[7] Cohen, M. L. and Bergstresser, T. K., Phys. Rev. 141 (1966) 789.

[8] Walter, J. P. and Cohen, M. L., Phys. Rev. 183 (1969) 763.

[9] For example an OPW calculation for $\mathrm{InSb}$ is given in Suratt, G. T. and Collins, T. C., Proc. XI Int. Semiconductor Conf. (Warsaw, 1972). An EPM calculation is given in Ref. 10.

[10] Varea de Alvarez, C., et al., J. Phys. \& Chem. Solids. (to be published).

[11] As another example, see the OPW calculation for CdTe by Herman, F. and Van DYKe, J. P., Section 10 of " Electronic Structure and Optical Spectrum of Semiconductors ", Aerospace Research Laboratories Report ARL 69-0080, Wright Patterson Air Force base, Ohio, 45433 (1969). An EPM calculation is given in Ref. 12.

[12] Chadi, D. J. et al., Phys. Rev. B 5 (1972) 3058.

[13] Phillips, J. C. and Pandey, K. C., Phys. Rev. Lett. 30 (1973) 790.

[14] CoHEN, M. L., private communication.

[15] SHaw, J., private communication.
[16] Horowitz, P. and Howell, J. A., Science 178 (1972) 608.

[17] This analyzer (Model 15-250) was built by Physical Electronics Industries, Edina, Minn.

[18] For several of the other semiconductors discussed in this paper, the $E_{\mathrm{F}}-E_{\mathrm{V}}$ separation is uncertain due to band bending (i. e. surface states). Consequently, we have determined $E_{\mathrm{v}}$ by extrapolating the high energy edge of the valence band PED to zero intensity and then placing $E_{\mathrm{v}}$ about $0.1 \mathrm{eV}$ below this point of zero intensity (the latter is meant to empirically account for our finite resolution).

[19] Eastman, D. E. and Grobman, W. D., Phys. Rev. Lett. 28 (1972) 1378.

[20] Ortenburger, I. B. and Rudge, W. E. (to be published).

[21] This shift also includes the band gap $\left(E_{\mathrm{c}}-E_{\mathrm{v}} \simeq 0.25 \mathrm{eV}\right)$; a situation which complicates interpretations of chemical shifts in semiconductors and in insulators.

[22] EPM density of states curves were kindly provided by CHAdi D. J. and COHEN, M. L. The following references contain the pseudopotential form factors used for individual semiconductors : Ge, Ref. $7 ; \mathrm{ZnSe}$ and GaP, Ref. 8 ; InSb, Ref. 10 ; CdTe, Ref. 12 ; GaAs, Ref. 23. In all cases the spin-orbit parameter was set equal to zero.

[23] Zucca, R. L., Walter, J. P., SHEN, Y. R. and Cohen, M. L., Solid State Commun. 8 (1970) 627. A pseudopotential coefficient $V_{\mathrm{s}}(8)=-0.008 \mathrm{Ry}$ was used rather than - 0.0008 which was a misprint in this reference.

[24] A similar broadening procedure has been used for all the EPM densities of states curves presented in this paper.

[25] For convenience we call the lower edges of the second and third bands in heteropolar semiconductors $X_{3}$ and $\Sigma_{1 \mathrm{~min}}$ respectively although the lower edge of the second band occurs at $L_{2}$ in some calculations, while the lower edge of the third band can be slightly removed from the minimum along $\Sigma_{1}$.

[26] Grobman, W. D., Eastman, D. E. and Cohen, M. L., Phys. Lett. (A) 43 (1973) 49.

[27] See also recent papers on $\mathrm{Si}, \mathrm{Ge}$ and CdTe by SARviA, L. R. and Casamajou, L., in : J. Phys. \& Chem. Solids (1971, 1972). 